\title{
ALGUNAS REFLEXIONES SOBRE LA OPERACIÓN DE REFLEXIVIZACIÓN
}

\author{
María Jesús Fernández Leborans \\ Universidad Complutense de Madrid
}

\begin{abstract}
RESUMEN
Este trabajo contiene discusión y argumentaciones en relación con recientes propuestas de análisis de las oraciones reflexivas. La discusión se centra fundamentalmente en tres aspectos: el denominado «diagnóstico de Reflexivización»; la naturaleza gramatical del clítico se y la derivación sintáctica de las oraciones reflexivas. Paralelamente, se argumenta en contra de la reducción argumental y de la conjunción de papeles temáticos en las oraciones reflexivas. Las diversas observaciones permiten concluir que la interpretación de Reflexividad oracional es sintácticamente, no léxicamente, inducida; no hay verbos reflexivos sino oraciones reflexivas.
\end{abstract}

Palabras clave: gramática teórica, sintaxis, semántica, verbos reflexivos, oraciones reflexivas, papeles temáticos, estructura argumental.

\section{Abstract}

This paper discusses recent analysis of reflexive sentences. The discussion is mainly concerned with three aspects of the issue: the diagnostics for the parameter setting with regard to reflexives, the grammatical nature of the $s e$ clitic, and the syntactic derivation of reflexive sentences. Furthermore, I argue against the argumental reduction and the bundling operation on $\theta$-roles in reflexive sentences. My observations allow us to conclude that the interpretation of sentential reflexivity is syntactically induced, and not lexically; there are not reflexive verbs, but reflexive sentences.

Key Words: Grammatical Theory, Syntax, Semantics, Reflexive Verbs, Reflexive Sentences, Theta-roles, Argumental Structure.

\section{INTRODUCGIÓN}

Uno de los aspectos de las construcciones reflexivas más debatidos en las últimas décadas en los estudios gramaticales tanto descriptivos como teóricos es el relativo a la propiedad de «intransitividad» que se atribuye a este tipo de construcciones, lo que resulta claramente inconsecuente con el valor de «transitividad» conferido a las oraciones reflejas (ORFL) por la tradición gramatical. La supuesta «intransitividad» de las ORFL ha sido considerada en los dos sentidos de «intransitividad»: la «ergatividad» o «inacusatividad» y la intransitividad propiamente di- 
cha o «inergatividad» ${ }^{1}$. En cualquier caso, las propuestas se fundamentan, entre otros razonamientos, en la caracterización del clítico reflejo se como una categoría morfológica no argumental (un morfema libre, no un pronombre genuino). Pues bien, este estudio contiene algunas reflexiones en relación con la propuesta de «inergatividad $»^{2}$; particularmente, se discute la denominada operación de "Reflexivización», propugnada por Reinhart y Siloni 2005, y se argumenta en contra de la consideración del clítico como mero recurso morfológico para la aplicación de la «Reflexivización» en la sintaxis de la oración reflexiva. Las argumentaciones que se aducen en este trabajo se fundamentan en la consideración de las oraciones reflexivas como oraciones transitivas que presentan correferencialidad argumental, tal como sanciona la tradición gramatical. El contenido de las páginas que siguen se distribuye en seis apartados: en el primero se incluyen contraargumentos respecto al denominado "diagnóstico de Reflexivización» y en el segundo se discute la supuesta reducción valencial en las oraciones reflejas, con atención particular a la naturaleza gramatical del clítico reflejo. El tercer apartado contiene observaciones sobre la conjunción de papeles semánticos en las oraciones reflexivas y en el cuarto se estudian contextos pasivos y de verbos Raising -verbos de «ascenso» o de «elevación de sujeto»- en relación con la Reflexividad. En el quinto apartado son objeto de discusión algunos aspectos sintácticos relativos a la derivación de las reflexivas y, por último, en el sexto se introduce un breve apunte en relación con las construcciones recíprocas.

\section{Sobre EL «Diagnóstico De ReFleXivización»}

La consideración de las oraciones reflexivas como oraciones intransitivas, «inergativas», parece inferirse de datos como los de (1a, b), construcciones que presentan un verbo con un solo argumento -el sujeto-; por analogía, las oraciones reflexivas de (1c, d) se analizan asimismo como un tipo de construcción inergativa con un solo argumento para un verbo que muestra la particularidad de aparecer con un clítico:

(1) a. John shaved (ing.)

b. Dan hitraxec (hebr.)

c. Pablo se afeitó

d. Paul se rase (fr.)

\footnotetext{
${ }^{1}$ Las propuestas favorables a la «inacusatividad» de las oraciones reflexivas surgen con anterioridad a las que propugnan la «inergatividad» de tales oraciones y, de hecho, cuentan con mayor número de defensores (v., entre otros, Bouchard 1984; Marantz 1984; Grimshaw 1990; Pesetsky 1995; Sportiche 1998).

${ }^{2}$ Las argumentaciones de Reinhart y Siloni 2004, 2005 en contra de la «inacusatividad» de las oraciones reflexivas son los suficientemente rigurosas como para no suscitar discusión al respecto.
} 
Puesto que los verbos de las oraciones reflexivas son naturalmente transitivos (son verbos de dos valencias: seleccionan dos argumentos -uno «externo» y otro «interno»-), alguna clase de reducción de argumento-valencia parece operar en tales construcciones; Reinhart y Siloni 2005 -desde ahora Reinhart y Siloni- proponen la Reflexivización, como operación que tiene que ver con la ausencia de un argumento-valencia y que puede aplicarse a la entrada léxica verbal o a la construcción sintáctica. Sin embargo, la «Reflexividad» no se obtiene en el Léxico, sino en la Sintaxis; no es una propiedad léxica, sino que ha de ser inducida por la sintaxis, aunque obviamente el significado del verbo ha de poder determinarla.

Digamos, entonces, que la correspondiente operación de reducción de valencia verbal se aplicaría exclusivamente en la sintaxis ${ }^{3}$. En este sentido, la propuesta fundamental de Reinhart y Siloni debe ser revisada; las autoras establecen un parámetro (The lex-syn Parameter) que distingue dos clases de lenguas: aquellas en las que la Reflexivización, como operación de reducción valencial, se aplica en el Léxico (Lexicon setting) y aquellas en las que tal operación se aplica en la Sintaxis (Syntax setting). Así, lenguas como hebreo, holandés, inglés, ruso, húngaro..., pertenecen al primer tipo (L-LEX) y lenguas como alemán, serbo-croata, griego, lenguas romances..., corresponden al segundo tipo (L-SINT). Sin embargo, el diagnóstico de "Reflexivización», que ofrece evidencias empíricas de esta diferenciación paramétrica ${ }^{4}$, no resulta del todo concluyente, como se observa a continuación.

Uno de los fenómenos que forman parte de este diagnóstico es la posibilidad de construcción con Predicativos con verbos del tipo considerar. Las lenguas SINT admiten tal posibilidad de modo generalizado, como puede observarse en (2), mientras que las lenguas Lex han de recurrir a pronombres anafóricos no propiamente reflejos para obtener interpretación paralela (pero sin operación de Reflexivización), como se muestra en (3):

(2) a. Peter se smatra pametnim. (serbo-croata)

b. Pedro se considera inteligente.

c. Paul se considère intelligent.

(3) a. *Dan mitxašev intiligenti. (hebreo)

\footnotetext{
${ }^{3}$ En algunas lenguas, como en español, existen verbos denominados por la tradición gramatical propiamente reflexivos, -o «pseudorreflejos» o verbos necesariamente pronominales- que son verbos lexicalizados con se (suicidarse, vanagloriarse, ufanarse, jactarse...), pero estos verbos, particularmente los afines a suicidarse no comportarían reducción de argumento ni siquiera en el léxico, por su valor -léxico- intransitivo (cf.: *Juan se suicidó a sí mismo; *Juan la suicidô), como se indica posteriormente. Por otro lado, también sobre la base del español, algún autor propugna la intransitividad de las oraciones reflexivas sobre la base de que el clítico no permite la expansión de un sN previsto en el léxico (véase Vera Luján 1996).

${ }^{4}$ Según el parámetro en cuestión, la Gramática Universal permite que las operaciones de reducción de valencia puedan ser aplicadas en el Léxico o en la Sintaxis. Los ejemplos del griego, ruso y serbo-croata recogidos en este trabajo son de Reinhart y Siloni 2005.
} 
Dan considera(REFL) inteligente.

b. Dan maxšiv et acmo intiligenti. (hebreo)

Dan considera $\mathrm{MO}^{5}$ a él mismo inteligente.

c. Dan considers himself intelligent. (ingl.)

Dan considera a él mismo inteligente.

Reinhart y Siloni arguyen que una operación léxica de Reflexivización no puede dar lugar a oraciones reflexivas de este tipo (ECM reflexives) porque la conjunción de dos papeles temáticos que implica tal operación ha de ser relativa a un mismo predicado, y en los contextos de este tipo hay dos predicados no relacionados en el léxico. Pues bien, dejando al margen que existen restricciones de selección léxica entre el predicado principal y el secundario (cf.: ? $\{$ me/lo\} considero de ojos azules), la conjunción de dos papeles temáticos se establece exclusivamente sobre la unicidad del referente en el contexto oracional, de modo que la Reflexivización como operación de reducción de valencia es cuestionable en tal caso. ¿Qué distingue las dos oraciones de (4), si no es exclusivamente la referencia disjunta en (4b)?; si admitimos que en (4b) el clítico (me/la) indica un argumento distinto del argumento sujeto del verbo considerar, no hay razón para rechazar que en (a) el clítico se marca asimismo un argumento distinto del argumento externo Juan, aunque con la particularidad, en este caso, de que ambos argumentos son correferentes:

(4) a. Juan se considera inteligente.

b. Juan $\{\mathrm{me} / \mathrm{la}\}$ considera inteligente.

Así que no se entiende bien por qué hay que aceptar que las lenguas supuestamente LEx que no admiten este tipo de construcciones MEC si no es con pronombres anafóricos, solamente inducirían Reflexivización semántica (interpretativa) en tales casos y las SINT presentarían Reflexivización sintáctica dado que contienen se, o una categoría similar ${ }^{6}$.

El segundo diagnóstico distingue las lenguas LEX de las SINT por la posibilidad de formación de sustantivos reflexivos (reflexive nominalizations) en el primer tipo pero no en el segundo. La explicación aducida es de carácter morfológico: se es incompatible con la morfología flexiva. Así, en las lenguas Romances no existen nominalizaciones reflejas, a diferencia de lo que ocurre en hebreo (5a) o en húngaro(5b):

(5) a. hitraxcut self-washing; a sí mismo-lavado

b. mos- akod -ás lavar-REFL-afijo nominal

${ }^{5} \mathrm{MO}$ («marca de Objeto») equivale a OM (object marker) de Reinhart y Siloni.

${ }^{6} \mathrm{Si}$ aceptamos, con Reinhart y Siloni, que la conjunción de papeles temáticos es uno de los fundamentos de la Reflexivización, ¿no resulta incongruente considerar que en las lenguas LEX no se da tal conjunción en los casos de construcciones con predicativo del objeto del tipo (a) cuando la interpretación de la oración es paralela a la de las construcciones análogas en las lenguas SINT? ¿Qué distingue la interpretación temática de (a) y (b)?:

a) Marie se considère intelligent.

b) Mary considers herself intelligent. 
Pero lo cierto es que en español formamos de modo productivo nominalizaciones reflejas con el prefijo auto: autocontrol, autoconocimiento, autodominio, autodestrucción, autocompasión, etc. Y estas formaciones no pueden ser consideradas sustantivos derivados de los correspondientes verbos reflexivos en el léxico, puesto que la mayor parte de tales verbos (controlar, conocer, dominar, destruir, compadecer...) no son léxicamente reflejos $^{7}$. Se trata de sustantivos en su mayor parte derivados de verbos transitivos causativos.

Un tercer diagnóstico establecido por Reinhart y Siloni es, asimismo, discutible. Las autoras consideran que en las lenguas SINT la Reflexivización es una operación productiva, mientras que, en las LEX , la Reflexivización se reduce a verbos cuyos argumentos ostentan los papeles temáticos Agente y Tema/Paciente. Pero la productividad de las L-SINT resulta ser solo aparente, dado que, en general, las oraciones en las que los papeles implicados son Causa o Experimentante no corresponden a construcciones propiamente reflexivas; no hay interpretación transitiva refleja natural en (6a): ? «Juan enfada \{a sí mismo / a él mismo)». Y, en cuanto a (6b), sí existe esa posibilidad, pero resulta ser excepcional (cf.: «Juan no se gusta nada a sí mismo» / ?? «Juan no se molesta / no se ofende / no se divierte / ...\} a sí mismo»).

(6) a. Juan se fache.

Juan se enfada.

b. Jean se plaît/déplaît sur cette photo.

Juan se gusta/disgusta en esta foto. («no se gusta en la foto»)

Por otro lado, los datos de lenguas de uno y otro tipo no permiten establecer una generalización sólida al respecto. Si bien podemos admitir que las L-LEX presentan verbos léxicamente reflexivos, que corresponden en su mayor parte a verbos transitivos de Agente-Paciente/ Tema, debemos reconocer que lenguas SINT presentan paradójicamente verbos «lexicalizados» exclusivamente con se que no dan lugar a construcciones propiamente reflejas. Así, en español, son numerosos los verbos de este tipo, tradicionalmente denominados verbos pseudo-

\footnotetext{
${ }^{7}$ Reinhart y Siloni consideran paralelamente construcciones con nominalizaciones del francés (a), perfectamente comparables con datos del español (b) relativas a verbos cuya interpretación inacusativa se obtiene via $s e$ :

a) le rétrécissement du pantalón au lavage (cf. le pantalón s'est retreci au lavage)

b) el \{alejamiento/ movimiento/desplazamiento/ aburrimiento/ enloquecimiento/ ...\} de María... (cf.: María se alejó/se movió/ se desplazó/ se aburrió/...)

La posibilidad de obtener «nominalizaciones» tiene que ver, según Reinhart y Siloni, con el hecho de que la formación de Inacusativos es una operación léxica incluso en las lenguas romances, es decir, se trata de una operación léxica en todas las lenguas. Sin embargo, los datos del español contradicen esta generalización; ciertamente, verbos como encoger, enloquecer,... son genuinamente inacusativos (cf.: el pantalón encogió; María enloqueció;...) pero otros muchos verbos susceptibles de formar «nominalizaciones» participan en la alternancia causativa (alejar, mover, acercar, desplazar, aburrir, molestar, disgustar,...), por lo que la interpretación inacusativa se obtiene en la sintaxis, en construcción con se. Así que la formación de «nominalizaciones» reflejas o «pseudorreflejas» (inacusativas) no siempre se deriva de entradas verbales léxicas correspondientes.
} 
rreflejos: jactarse, suicidarse, ufanarse, vanagloriarse, etc. Sin embargo, tales verbos, y también los que, como en (6), presentan alternancia con la forma no pronominal e intervienen naturalmente en construcciones «pseudorreflejas», permiten derivar eventos inergativos o inacusativos con un único argumento (con el papel de Agente en el primer caso y de Tema/Experimentante en el segundo). Diferentemente, los eventos de interpretación propiamente reflexiva no implican reducción a un único argumento, sino correferencialidad de dos argumentos, de modo que las propuestas relativas a la reducción de valencia-argumento inherentes a la Reflexivización han de ser revisadas.

\section{Contra la Reducción VAlencial EN LA ReFLeXivización}

A partir del supuesto de que el léxico es activo (Siloni 2002) y permite, por tanto, la aplicación de operaciones derivacionales, previas a la inserción sintáctica, la propuesta de Reinhart y Siloni 2005 determina la Reflexivización como una operación que afecta a la valencia interna -argumento interno- de un verbo transitivo, de modo que permite obtener verbos reflexivos, esto es, verbos que denotan acciones que el argumento Agente realiza sobre sí mismo o estados mentales que el argumento Experimentante adquiere con respecto a sí mismo, sin realización de un argumento objeto reflexivo, como en (7). La interpretación reflexiva puede lograrse también mediante expresiones anafóricas reflejas, como en (8) -ambos son ejemplos del hebreo presentados por Reinhart y Siloni-:

(7) Dan hitraxec.

Dan lavó(REFL).

(8) Dan raxac et acmo.

Dan lavó a él / sí mismo.

Los verbos reflexivos presentan particularidades morfológicas: un afijo verbal determinado (en hebreo, en ruso, cf. 9a, etc.); un clítico (en las lenguas romances: $s e$, francés, español, italiano, serbo-croata, etc. (cf. 9b), o morfología cero (como en inglés (9c), por ejemplo) o en euskera (9d):

(9) a. Ona pomylas'.

Ella lavó(REFL).

Ella se lavó

b. Marie s'est lavé. / María se ha lavado. / María si lavava. / On se oprao.

c. John washed.

John se lavó.

d. Garbi-tuda

limpiar-PERF Aux.3N

Él se limpió

\footnotetext{
${ }^{8} \mathrm{~N}$ indica concordancia de Nominativo. En euskera, el uso intransitivo de los verbos, como en (9d), puede tener, además de la lectura refleja, la interpretación pasiva («\{él / eso\} ha sido limpiado»). V. Wunderlich 2006.
} 
La Reflexivización, entendida como operación universal -las denominadas Arity Operations, es decir, las operaciones que afectan a la valencia de un predicado son universales- puede aplicarse en el Léxico o en la Sintaxis, lo que permite establecer, como se mencionó anteriormente, una variación paramétrica (lex-syn parameter). Ahora bien, la motivación que induce a Reinhart y Siloni a establecer el parámetro Léxico-Sintaxis con respecto a la Reflexivización no resulta concluyente ni empírica ni conceptualmente en relación con la propia operación de Reflexivización. La discusión tiene que ver con dos aspectos importantes: en qué consiste tal operación y cuál es la naturaleza de los clíticos del tipo se. Discutimos a continuación este último aspecto.

3.1. Reinhart y Siloni afirman que el clítico se/si de las lenguas romances forma verbos reflexivos, y siguiendo observaciones de Kayne 1975 consideran que tal clítico no posee función argumental, no es un objeto clítico de un verbo transitivo. De modo que los verbos con se no se comportarían como verbos transitivos; Kayne 1975 aduce, para el francés, dos pruebas, basadas en dos tipos de contextos que ponen de manifiesto el comportamiento «intransitivo» de los verbos con se. El primer tipo de contexto es el de inserción de expletivo: los verbos intransitivos, pero no los transitivos, admiten este contexto, cf. (10a) y (10b), y también los verbos con se aceptan este contexto (10c), si bien no es unánime la aceptabilidad:

(10) a. Il est arrivé trois filles.

b. *Il les a dénoncés $t_{\mathrm{i}}$ trois mille hommes ce mois-ci.

c. ? Il s'est dénoncé trois mille hommes ce mois-ci.

Pero lo cierto es que la prueba no resulta ser determinante; en el caso de (10a), se trata de verbos inacusativos, no inergativos (intransitivos puros). Y dejando al margen la inconveniencia de un ejemplo como (10b) en el conjunto de la argumentación que se discute, porque no prueba adecuadamente la incompatibilidad de los verbos transitivos con la inserción de expletivos ${ }^{9}$, no es obvio que (10c) muestre una construcción propiamente reflexiva -sin interferir con el valor impersonal reflejo- como indica su relativa aceptabilidad ${ }^{10}$.

Bien sabido es que en el origen de todas las formas verbales pro-

\footnotetext{
${ }^{9}$ De hecho, los verbos transitivos requieren, además del argumento interno directo, un argumento externo, es decir, un sujeto referencial, lo que implica incompatibilidad con un sujeto expletivo. Casos excepcionales son los de construcción defectiva de sujeto con verbo transitivo como il fait froid, etc., es decir, cierto tipo de construcción «impersonal».

${ }^{10}$ Los juicios de gramaticalidad de los hablantes nativos consultados, coinciden en asignar la interpretación impersonal refleja a la construcción (10c), con el sentido de «se ha denunciado a tres mil hombres este mes»; «tres mil hombres han sido denunciados...». (El juicio de aceptabilidad dudosa aparece en el texto de Reinhart y Siloni; las autoras indican en nota (p. 392) que, si bien para Kayne 1975 la mencionada construcción es gramatical, se trata, para algunos hablantes, de una construcción marginal).
} 
nominales está el se clítico reflexivo, y que tal clítico ha perdido su estatus nominal de objeto argumental en las diversas construcciones no propiamente reflexivas, pero ¿por qué habríamos de negarle su estatus argumental en las reflexivas? ¿qué distingue los clíticos de (11a) y (11b) si no es solamente la correferencialidad en (11b)?:

(11) a. Pierre ${ }_{\mathrm{i}} \mathrm{le}_{\mathrm{j}}$ lave

b. Pierre ${ }_{\mathrm{i}}$ se $_{\mathrm{i}}$ lave.

En este aspecto, los datos del español -y de otras lenguas romancesson similares: claro es que el estatus funcional de $l a$ y de $s e$ o de $m e$, en (12) es exactamente el mismo:

(12) a. María la peina

b. María se peina

c. Yo me peino

Por lo que respecta a la segunda prueba aducida por Kayne 1975, no parece cuestionable. Se basa en las construcciones causativas del francés, las cuales, según Kayne, permiten asociar el comportamiento de los verbos reflexivos con los intransitivos; ambos introducen el sujeto de los verbos subordinados al causativo faire sin la preposición $\grave{a}$-como se puede observar en (13a, b) - a diferencia de lo que sucede con los transitivos, cuyo sujeto semántico es introducido por esta preposición (13c). Yen un sentido análogo, Reinhart y Siloni observan que la diferente posición de los clíticos pronominales y reflexivos en (13d) y (13e) sugiere comportamientos sintácticos distintos:

(13) a. Je ferai courir Paul.

b. Je ferai se laver Paul.

c. Je ferai laver Max*(à) Paul.

d. Je le ferai laver à Paul.

e. Je ferai se laver Paul.

Pero en este caso, la observación es imprecisa, porque se trata de construcciones distintas en lo que a la «cliticización» se refiere; la posición diferente de los clíticos en (13d) y en (13e) tiene que ver con el hecho de que el clítico reflejo no puede presentar proclisis al verbo causativo en francés, y tampoco esta posibilidad está disponible en español, como puede observarse en (14e) -a partir de (14d) - . Los datos del español permiten observar similitud entre verbos «reflexivos» y verbos intransitivos (14d, f), y disimilitud con los transitivos (14a-c), también en lo que respecta a la posición de los clíticos en los mismos contextos de verbos causativos:

(14) a. Le haré lavar la bicicleta a Pablo.

b. Le haré lavarla.

c. Yo se la haré lavar $(s e=l e)$.

d. Yo $\{$ le/?la $\}$ haré lavarse (a María) ${ }^{11}$.

${ }^{11}$ Los juicios de gramaticalidad-aceptabilidad de los hablantes difieren respecto al uso del 
e. *Yo se se haré lavar $(s e=l e)$.

f. \{?Le/ la\} haré sonreír (a María).

g. Le haré lavarme.

h. *Se me haré lavar.

Pero ha de tenerse en cuenta, una vez más, que la diferencia de posición tiene que ver con la distinta naturaleza de los clíticos; se ha observado acertadamente que los clíticos de Persona Tercera no reflejos ostentan comportamientos sintácticos distintos de los de Persona Primera, Segunda y propiamente reflejos (Anderson 2005); obsérvese que tampoco podemos construir (14h), con clítico de objeto directo no reflejo, a partir de $(14 \mathrm{~g})^{12}$. Y, además, precisamente los de concordancia de Objeto directo de referente animado, específicamente personal, ofrecen resistencia a la proclisis en contextos causativos -y otros contextos de Reestructuración de verbos- a diferencia de los de referente no animado. (Cf.: le hizo examinar los documentos (a Maria) $\rightarrow$ se los hizo examinar; el director le hizo examinar a los dos alumnos (a Maria) $\rightarrow$ *?se los hizo examinar; le vi coger el sobre (a Pedro) $\rightarrow$ se lo vi coger; le vi acostar a la niña a Pedro $\rightarrow{ }^{*}$ se la vi acostar $)^{13}$.

Así que no parece que haya pruebas definitivas para concluir que el $s e$ de las contrucciones reflexivas con verbos transitivos sea un marcador de reflexividad léxica -como propone Chierchia 2004-; se trata de un clítico indicador de función argumental de objeto, como se ha destacado más arriba, o, si se prefiere, un marcador de concordancia de objeto. Pero, respecto a la forma $s e$, es precisamente su subespecificación lo que ha permitido su lexicalización como marca o morfema portador de otros valores, incluido el reflexivo lexicalizado. Un verbo como suicidarse diríamos que es reflexivo (morfológicamente) en el léxico, pero no en la sintaxis; no puede constituir oraciones propiamente reflexivas, porque es monoargumental ${ }^{14}$. Y, correlativamente, no diríamos que un verbo como lavar es reflexivo en el léxico, pero permite formar oraciones reflexivas con un argumento objeto que es correferencial con el argumento externo sujeto y que está indicado por se o alguna otra forma del paradigma ( $m e, t e, n o s$, os). Solamente esta proforma se (o forma análoga

clítico de acusativo en (14d) y en (14f). A diferencia de lo que sucede en (14d) -con reflexivos- en construcciones del tipo (14f) el clítico acusativo es la opción preferida por numerosos hablantes. Ello sugiere que el clítico no reflejo de (14d) se siente gramaticalmente como un dativo porque hay un clítico reflejo de acusativo.

${ }^{12}$ Estas construcciones pueden considerarse como una manifestación más del efecto de la denominada «me-lui constraint» (desde Perlmutter 1971). La restricción establece que un clítico pronominal dativo no puede coaparecer con un clítico acusativo de \{Primera/Segunda\} Persona. Cf.: *tú se me enviaste a Juan / tú se la enviaste a Juan. (V. Sáez 2007).

${ }^{13}$ Véase al respecto, Cano 1987 y Sáez 2007.

${ }^{14}$ Obsérvese -como ha señalado reiteradamente la tradición gramatical- que solamente la construcción propiamente reflexiva admite expresiones anafóricas léxicas, plenas, como en: Ana se quiere mucho a sí misma; por el contrario, la agramaticalidad de *Ana se suicidó/ se vanagloria / se jacta/...\} a sí misma se sigue del carácter monoargumental del verbo en el léxico. 
en las lenguas romances) es el clítico totalmente subespecificado, en el sentido de que no contiene especificación de persona -su rasgo es de $3^{\text {a }}$ persona, que es la no marcada o «no-persona»- ni de número -el rasgo de número presenta sincretismo de singular y plural-. Y, dada su subespecificación, es esperable su disponibilidad para ser dotado con algún tipo de rasgo que tiene que ver, en general, con la «desactivación» de un argumento o papel temático en relación con la «reducción» de la estructura subeventiva de un núcleo predicativo o, simplemente, con la «neutralización»-indeterminación- de algún argumento por su irrelevancia informativa. Así, la inacusatividad o ergatividad de «causa externa» de el vaso se rompió induce inoperatividad del argumento "Agente/Causa", porque importa el subevento relativo al estado resultante que afecta al objeto. Y en la pasividad impersonal de se hacen fotocopias se neutraliza el argumento Agente (por indeterminación -impersonalidad, en la tradición gramatical) ${ }^{15}$.

3. 2. En cuanto a la operación de Reflexivización, que puede ser léxica o sintáctica, según parámetro, como se ha mencionado más arriba, su definición como reducción de valencia de un predicado, puede ser contestada en más de un aspecto ${ }^{16}$. En primer lugar, según Reinhart y Siloni, la operación de Reflexivización en las lenguas SINT determina «inergatividad sintáctica», es decir, el verbo en las construcciones reflexivas de las L-SINT es un verbo sintácticamente inergativo, de una valencia, porque la Reflexivización permite que un papel temático no se asigne a la posición de argumento sintáctico correspondiente, aun cuando la semántica del verbo y la interpretación de la oración mantiene la red temáticoargumental completa original de la entrada léxica básica transitiva. Así que la reducción de una valencia en la sintaxis no se corresponde con una reducción en la semántica léxica de un papel temático; para Reinhart y Siloni, la Reflexivización no sería propiamente una operación de reducción de valencia ${ }^{17}$, sino de composición o «atado» (bundling) de dos papeles temáticos para formar un papel temático complejo. Consecuentemente, el efecto de la operación RFLX es -parafraseando a R.-Sque dos papeles temáticos (p. ej. Agente y Paciente/Objeto Afectado) son asignados al mismo argumento sintáctico. Correlativamente, la ope-

\footnotetext{
${ }^{15}$ Chierchia 2004 emplea el término decausativization para el primer caso, y habla de saturation en el segundo.

${ }^{16}$ Las argumentaciones desarrolladas por Reinhart y Siloni 2005 respecto a la inadecuación de la derivación inacusativa para las construcciones reflexivas son incuestionables, por lo que las observaciones que se desarrollan a continuación en el texto cuestionan fundamentalmente la supuesta inergatividad sintáctica de los verbos reflexivos tanto en las lenguas L-LEX como en las L-SINT.

${ }^{17}$ La hipótesis de la reducción de valencia para las reflexivas, entre otras construcciones, ha sido defendida fundamentalmente por Chierchia 2004. Y, en realidad, las propuestas que presentan las construcciones reflexivas como inacusativas asumen de un modo u otro la reducción valencial.
} 
ración RFLX ha de comportar reducción de Caso, dado que el verbo transitivo básico al que se aplica tal operación posee un rasgo de Caso acusativo que requeriría cotejo. Pues bien, si dejamos al margen, por el momento, este último aspecto de la reducción del rasgo de Caso, intuitivamente resulta algo arbitrario hablar de «inergatividad» sintáctica, porque la inergatividad -como la «inacusatividad» o la «transitividad»- es una propiedad de la semántica léxica de los núcleos predicativos; ¿diríamos que (15a), por ejemplo, presenta también inergatividad sintáctica como (15b)?; ¿ ¿sería asimismo sintácticamente inergativa la construcción (15c)? No parece que sea preciso demostrar que respuestas afirmativas a estas cuestiones serían contraintuitivas:

(15) a. Ana las lavó.

b. Ana se lavó.

c. Te lavaste.

Los clíticos, en cualquier caso análogos de las construcciones de (15), presentan marca de función, marca de Caso y remiten a un argumento portador de papel temático «interno»; pero en el caso de los clíticos reflejos, la concordancia de rasgos nominales (de Persona, Número y Género) del clítico con los que se afijan a la correspondiente forma verbal (los denominados morfemas nominales flexivos del verbo) hace innecesaria la realización fonética - la «visibilidad» fonética- del argumento Objeto, de modo que tanto si postulamos una categoría vacía (pro) correferencial con el clítico como si no, el clítico permite reconocer, identificar, un argumento Objeto que correfiere con el argumento Sujeto. (Paralelamente, el clítico no reflejo -como en (15a)-indica un argumento Objeto no correferencial con el argumento Sujeto.)

Por lo que respecta a las lenguas LEx, la operación RFLX se aplicaría, según Reinhart y Siloni, a la entrada léxica transitiva, de forma que, por ejemplo, a partir de una entrada léxica como wash, marcada con el rasgo de Caso acusativo y con las dos valencias-argumentos correspondientes -véase (16a) - se obtiene, mediante la aplicación de RFLx, otra entrada wash refleja, esto es, con reducción de Caso acusativo y conjunción de los dos papeles correspondientes en un solo argumento, que es el externo, como se indica en (16b); RFLx opera antes de la derivación sintáctica (16c). Técnicamente, la representación semántica se muestra en $(16 \mathrm{~d})$, y ha de ser interpretada como una «conjunción distributiva» de papeles temáticos (16e):

(16) a. wash ac [Agente] [Tema]

b. wash [Agente-Tema]

c. Sintaxis: Max[Agente-Tema] washed

d. [wash (e) [Agente-Tema] (e, Max)]

e. [wash (e) Agente (e, Max) Tema (e, Max)]

Pero esta clase de interpretación que Reinhart y Siloni atribuyen a 
la composición de papeles temáticos es incompatible con la hipótesis del papel temático complejo asignado a un único argumento, que es el externo. El carácter distributivo implica, por principio, «desdoblar» el argumento; indiscutiblemente, disponemos de un solo referente en la semántica de la predicación refleja, pero seguimos teniendo dos argumentos que son correferenciales ${ }^{18}$. En lenguas del tipo L-LEx que no disponen de marcas de reflexividad, se infiere sin dificultad la lectura refleja en oraciones como Max washed, simplemente porque la interpretación por defecto que se sigue en ausencia de proformas de argumento Objeto, como it, him, etc., de referencia disjunta $-\mathrm{o}$, naturalmente, sin argumento Objeto pleno, sintagmático, realizado fonéticamente, como the child, etc.- es precisamente la reflexiva, sintácticamente inducida por la derivación argumentalmente defectiva de la oración con verbo transitivo ${ }^{19}$. Así que, en cualquier caso, la reflexividad resulta ser un fenómeno de aparente «intransitividad sintáctica» derivada de transitividad léxica con argumentos Agente y Tema/Paciente correferenciales, por lo que no es requerido un AI explícito (fonéticamente visible).

\section{4. «REFLEXIVIZACIÓN» Y «COMPOSICIÓN» DE PAPELES TEMÁTICOS}

Un aspecto particularmente cuestionable en la operación de Reflexivización, es el requisito de la «conjunción» (bundling) de papeles temáticos; tal conjunción debe operar sobre un papel temático «externo», es decir, un papel que obligatoriamente se ensamble en posición externa (merges externally), por ejemplo, un Agente, como se representa en (17):

\section{(17) Reflexivization bundling}

$[\theta \mathrm{i}][\theta \mathrm{j}] \rightarrow[\theta \mathrm{i}-\theta \mathrm{j}]$ donde $\theta \mathrm{i}$ es un papel- $\theta$ externo

Ahora bien, ciertamente la clave de la interpretación refleja es el papel temático externo, asociado a un argumento externo (AE). El hecho de que en la sintaxis se pueda obviar el argumento interno (AI), que, sin embargo, sigue siendo accesible en la semántica, tiene mucho que ver con el AE receptor de un papel temático que ha de observar una restricción: debe ser un papel temático asociado al rasgo [+animado] y, específicamente [+humano]. Así que, en las llamadas lenguas L-LEX, el Ensamble de una raíz (root) verbal V con un afijo «reflejo» (como en hebreo), y/o con un argumento fonéticamente nulo (como en inglés), inducirá la interpretación reflexiva (correferencialidad de argumentos)

\footnotetext{
${ }^{18}$ Dicho de otro modo, la atribución a un mismo referente de dos papeles temáticos, Agente y Tema, implica otorgarle función biargumental necesariamente.

${ }^{19}$ Una derivación defectiva análoga puede recibir interpretación de evento atélico de «Actividad», y en tal caso, solamente el contexto previo o la situación de comunicación permitirán decidir la lectura adecuada.
} 
de la derivación, mientras que en las consideradas lenguas L-SINT, tal interpretación se seguirá del Ensamble con el clítico se (o similar, como en español) o con una expresión anafórica correferencial. Se habrá advertido, tras esta observación, que, de hecho, ya no parece pertinente la distinción LEX-SINT ¿Por qué habría de ser distinto el Ensamble con afijos «trabados» del Ensamble con clíticos o afijos «libres»? Se trata, en cualquier caso, de relaciones entre categorías del léxico. ¿Acaso es distinta la relación entre el Determinante y el Nombre cuando aquel es un afijo de este o una categoría morfológicamente independiente?

Pero, además, lo que quiero proponer es que la condición del AE ha de ser observada para la interpretación reflexiva en la Fase $v^{*}$ de modo que el Ensamble del Sv con esta categoría funcional $v^{*}$ permite en cualquier lengua la interpretación reflexiva, que no puede implicar reducción valencial, a diferencia de lo que ocurriría, por ejemplo, con una variante de interpretación inacusativa, inducida por Ensamble con una categoría funcional v. Así, lo que distingue la oración (18a) de la (18b) es el hecho de que, en la primera, la proyección inmediatamente superior al Sv es $S v^{*}$, mientras que, en la segunda, es $S v$. Como es sabido, la proyección $S v$, con $v$ simple, no constituye Fase, de modo que el AE Juan en (18b) es, en realidad, derivado de un Objeto (AI) por lo que la proyección $S v$ es defectiva y no se transfiere al c.I-C (Componente Intencional-Conceptual) hasta que se alcanza en la derivación la Fase sc (Chomsky 2006). Diferentemente, en (18a), ya se genera Juan como AE en el Especificador de $S v^{*}$, que constituye Fase transferible al C.I-C porque la categoría $v^{*}$ encabeza sintagmas verbales de estructura argumental completa (con verbos transitivos e inergativos) ${ }^{20}$. (Las dos proyecciones se ilustran en (19a) y (19b) respectivamente).

(18) a. Juan se mató (con la escopeta de caza). (interpretación transitiva reflexiva)

\footnotetext{
${ }^{20}$ Como se indica en repetidas ocasiones en varios estudios de Chomsky pertenecientes al denominado Programa Minimista, los verbos transitivos e intransitivos (inergativos) se extienden en proyecciones del tipo $S v^{*}$, mientras que los inacusativos y pasivos lo hacen en proyecciones del tipo $S v$. La categoría funcional $v$ *tiene que ver con estructura argumental «completa», de carácter proposicional -considera Chomsky- lo que implica argumento externo, a diferencia de la categoría $v$, relativa a la inexistencia de un argumento externo.
} 
b. Juan se mató (en un accidente de automóvil). (interpretación inacusativa_)

(19) a.
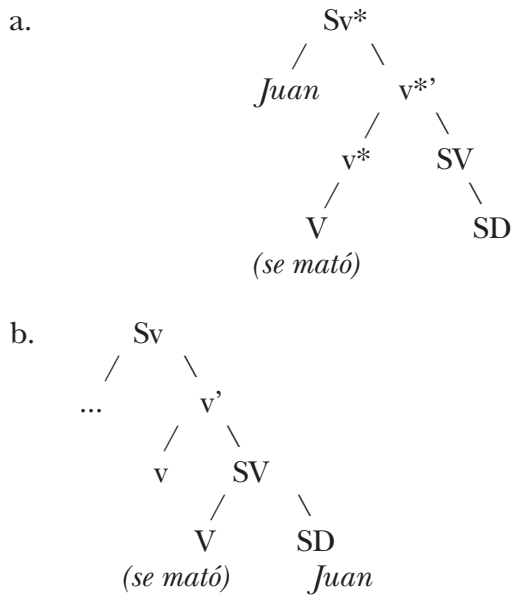

Si tales consideraciones son correctas, resulta que, de hecho, la diferencia entre $v^{*}$ y $v$ tiene que ver con la propiedad [+AE] y [-AE] respectivamente, con la particularidad, además, de que $v^{*}$, pero no $v$, comporte un «rasgo» de Caso estructural Acusativo. Desde este punto de vista, y teniendo en cuenta, subsidiariamente, que la categoría funcional $v^{*}$ / $v$ determinaría el carácter verbal de la raíz ${ }^{21}$, por lo que esta no sería portadora de ningún rasgo propiamente gramatical o «computacional», podríamos entender que el rasgo no-interpretable de Caso estructural Acusativo es una propiedad de $v^{*}$, y que su asignación debe realizarse dentro de la proyección $S v^{*}$ (Chomsky 2006) ${ }^{22}$. Por otro lado, si asociamos la propiedad $[+\mathrm{AE}]$ a $[+$ Papel $-\theta \mathrm{E}]$ podemos recoger sin dificultad la clásica Generalización de Burzio 1986 en el sentido unidireccional -no biunívoco- que interesa:

(20) No se puede asignar Caso estructural al AI, si no se asigna Papel Temático al AE.

Y, probablemente, podría tomarse en consideración la posibilidad de que ambas categorías funcionales $v^{*}$ y $v$ estén asociadas a determinados papeles temáticos, es decir, que la asignación temática que corresponde al C.I-C, esté «guiada» por cualquiera de estas categorías. Dicho de otro modo, si todos los papeles temáticos son accesibles en la proyec-

\footnotetext{
${ }^{21}$ En la línea de las propuestas de Marantz 1997, 2005 y seguidores.

${ }^{22}$ En el texto de Chomsky 2006 se establece que los rasgos no interpretables de Caso estructural y Acuerdo o Concordancia redundante, deben ser validados en configuraciones sintácticas: el Caso Nominativo estructural y Concordancia se asignan dentro de la proyección SC (T hereda los rasgos de C) y el Caso acusativo estructural y concordancia de Objeto se asignan dentro de $\mathrm{Sv}^{*}$.
} 
ción inmediatamente superior a $\mathrm{SV}^{23}$ parece que las categorías mencionadas tienen algo que ver con la asignación temática en la interfaz I-c; así, el AE en la posición de [Espec, Sv*] induciría la interpretación en la interfaz I-C de ciertos papeles temáticos y no otros, en relación, además, con la existencia o no de un $\mathrm{AI}^{24}$. Lo que quiero destacar -sin introducir discusión aquí- es que, plausiblemente, las relaciones temáticas estén de algún modo codificadas por las categorías funcionales $\left(v^{*} / v\right)$ con las que se ensambla directamente una raíz verbal en la derivación ${ }^{25}$.

Volviendo a las construcciones reflexivas, la presencia requerida del AE en tales construcciones -como en las transitivas en general- está asegurada por la categoría $v^{*}$ con la que el $\mathrm{V}$ se ensambla, de modo que no es preciso suponer que la red temática del V léxico incluye información sobre el «papel temático externo» como asumen Reinhart y Siloni. Cabe otra precisión: debe distinguirse adecuadamente entre «Papel Externo» y «Argumento Externo». No hay razón para considerar que la entrada léxica verbal contenga información sobre posiciones «externas» o «internas», a menos que se ensamble con una categoría funcional; así, el Argumento Externo que habilita la categoría funcional $v^{*}$, podrá ser asociado con un determinado papel temático, como se ha observado anteriormente, que será, consecuentemente, un papel «externo». Obsérvese, sin embargo, que el carácter "externo" de un papel temático es relativo, en el sentido de que tiene que ver con su relación con el papel del $\mathrm{AI}^{26}$, cuando se trata de verbos con dos argumentos, etc. En cualquier caso, los papeles temáticos «candidatos» a la posición externa (como Agente, Experimentante...) -así como los «candidatos» a la posición interna (como Paciente, Tema...) - podrían estar inducidos por

${ }^{23}$ Tal como observa Chomsky 2006. No obstante, no está del todo claro cómo es asignada la estructura argumental, la relación semántica entre núcleo predicativo y $\operatorname{argumento(s),~tal~}$ como el propio Chomsky 2005, 2006 indica.

${ }^{24}$ Considérese, por ejemplo, que el argumento de un $\mathrm{V}$ asociado a una categoría funcional $v$-un verbo ergativo- no es, por defecto, portador de un papel temático de Agente, etc.

${ }^{25}$ Una propuesta original en este sentido es la que defiende Arellano 2008.

${ }^{26}$ Como indica Gruber 2000, se ha establecido, al respecto, un orden generalizado de precedencia relativa, una jerarquía temática. Básicamente, se ha establecido la relación jerárquica entre posiciones externas (a) y posiciones internas (b) -asimetría «sujeto/objeto»- como correlativa de la siguiente distribución generalizada de papeles temáticos:

(a)

$\begin{array}{lll}\text { (sujeto) } & \text { (b) } & \text { (objeto) } \\ \text { Agente/Causa } & & \text { Paciente/Tema } \\ \text { Experimentante } & & \text { Tema } \\ \text { Instrumento } & \text { Tema } \\ \text { Fuente } & \text { Tema } \\ \text { Locación } & \text { Tema } \\ \text { Tema } & & \text { Meta }\end{array}$

La jerarquía temática presentada se interpreta del modo siguiente: dado un predicado léxico que selecciona dos argumentos, uno con el papel de Agente y otro con el de Tema, el primero se realizaría como sujeto sintáctico y el segundo como objeto sintáctico; dado un predicado léxico que selecciona un argumento Experimentante y un argumento Tema, el primero se corresponderá con el sujeto sintáctico (posición externa) y el segundo con el objeto (posición interna), etc. (v. Fernández Leborans 2005). 
las categorías funcionales $v^{*}, v$, facilitando así su interpretación por el C. I-C.

Pero la «conjunción» de papeles temáticos no sería consistente con estas observaciones; bundling no puede ser aplicado directamente a la red temática del verbo - the verb's grid- en las lenguas LEX sencillamente porque la única razón para esta operación de conjunción de papeles es la correferencialidad, y esta relación -la referencia en general- no pertenece al Léxico, sino al C.I.C ${ }^{27}$. Un razonamiento paralelo puede argüirse en el caso de las lenguas SINT, esto es, cuando se considera que bundling se aplica en la sintaxis; Reinhart y Siloni proponen que bundling sintáctico se aplica, no a la red temática del verbo, sino a papeles no asignados, de modo que, cuando se ensambla el AE en la sintaxis, este argumento se convierte en un bundle of roles. El papel del AI es retenido en la proyección verbal hasta que el papel externo se ensambla en la estructura sintáctica, y mediante la operación indicada en (17), el AE, único argumento sintáctico, recibe dos papeles temáticos. Pero, nuevamente, los hechos pueden ser considerados de otro modo. Ciertamente, el papel del AI no puede ser asignado en su posición canónica, a menos que se propugne una $\mathrm{CV}$ - una categoría vacía ${ }^{28}$ - correferencial con el clítico y/o con el AI, como receptora de tal papel temático. En cualquier caso, no habría asignación de Caso acusativo estructural, pero, como se ha indicado más arriba, el clítico posee Caso morfológico acusativo -lo que implica hablar de «reducción» del rasgo de Caso estructural- pero ¿por qué esta marca de Caso morfológico no puede estar asociada de algún modo con una marca de Papel Temático correspondiente al AI? Si es así, el sistema I-C puede acceder sin dificultad a la interpretación de correferencialidad, obviándose todo tipo de operaciones «extra» del tipo (17), que no están lejos del carácter estipulativo ad hoc.

En determinadas lenguas LEX, la interpretación de correferencialidad argumental puede seguirse con un AI no realizado - no visiblefonéticamente cuando el verbo expresa acciones físicas que inducen lectura refleja naturalmente (acciones realizadas por un agente sobre su cuerpo, como afeitarse $)^{29}$; en otro caso, precisan de la expresión de una proforma correferencial; cf. las dos construcciones de (21):

\footnotetext{
${ }^{27}$ Chomsky 2006 es claro al respecto: «[...] there is no reference-like relation for human language, hence no semantics in the technical sense of Frege, $[\ldots]$ ».

${ }^{28}$ En el marco del modelo PP (de «Principios y Parámetros») o RL (de «Rección y Ligamiento»), se trataría de una categoría vacía del tipo pro argumental de Objeto, legitimada por el papel Temático que debe ser asignado y habilitada en el contexto de Rección por $\mathrm{V}$ (Chomsky 1982; Rizzi 1986); en el marco del PM (Programa Minimista) no parece haber razón teorética alguna para «cancelar» este tipo de categoría.

${ }^{29}$ Como se ha observado en más de una ocasión, son precisamente los verbos que expresan acciones de naturaleza física realizadas por un Agente sobre su cuerpo o en relación con su integridad física los que se codifican sin dificultad en el léxico como verbos reflexivos en algunas lenguas (LEX), con o sin afijos reflexivos, por ejemplo, los verbos del hebreo correlativos de afeitarse, lavarse, equiparse, armarse, etcétera.
} 
(21) a. John shaved.

b. *John loves / John loves himself.

No obstante, la ausencia del AI en la derivación no es algo que precise explicación adicional; la interfaz semántica (el componente I-C) nunca asignaría interpretación propiamente inergativa a un evento como el de (21a), porque ello conllevaría reducción de un papel temático, el del AI. Y de nuevo insistimos en que las construcciones reflexivas no comportan reducción de papel temático.

\section{Reflexividad en contextos de Construcción Pasiva y Construc- CIÓN CON VERBOS DE «ASCENSO»}

Interés particular presentan las construcciones de (22) que muestran, aparentemente, una incompatibilidad entre Reflexividad y construcción pasiva / construcción Raising (con verbos de «Ascenso») ${ }^{30}$ :

(22) a. *Gianni si è stato affidato.

b. *Jean se semble intelligent.

c. *?Juan se parece inteligente.

Las construcciones resultan aceptables con expresiones pronominales anafóricas in situ:

(23) a. Gianni si è stato affidato a se stesso.

b. Jean ne semble intelligent qu'à lui-même.

c. Juan se parece inteligente a sí mismo (a los demás no se lo parece $)^{31}$.

Por otro lado, las construcciones inaceptables de (22) son perfectas con clíticos no correferenciales:

(24) a. Gianni gli è stato affidato.

b. Jean leur semble intelligent.

c. Juan me parece inteligente.

La aparente incompatibilidad se ha esgrimido como un argumento empírico a favor de la derivación inacusativa de los verbos reflexivos. Según estas argumentaciones, el clítico reflexivo se «absorbe» el papel externo -en el léxico o en la sintaxis-, de modo que no habría papel externo disponible para los predicados relevantes de las construcciones de (22). Pero esta consideración es, a mi juicio, errónea, porque la «absorción» del papel externo también se daría en las construcciones paralelas de (23), con las proformas anafóricas expresas. Desde otra perspectiva

\footnotetext{
${ }^{30}$ Este fenómeno ha sido estudiado, entre otros, por Kayne 1975; Rizzi 1986; Burzio 1986. Los ejemplos citados están tomados de Reinhart y Siloni.

${ }^{31} \mathrm{El}$ juicio de los hablantes consultados es de aceptabilidad total para construcciones como las de (a), sin el requisito de la expresión anafórica:

a)Tú siempre te pareces \{poca cosa / un ser mediocre / la persona más lista del mundo\} (a ti misma)
} 
distinta a la de la inacusatividad, como es la de la inergatividad de las construcciones reflexivas, se ha explicado la supuesta incompatibilidad de (22) de modo no muy diferente. Así, Reinhart y Siloni 2005 la infieren de la imposibilidad de satisfacer la operación de Reflexivization Bundling aplicada en la sintaxis en las lenguas SINT. Esta operación se aplica a papeles temáticos no asignados, en la posición de Ensamble del papel temático externo. Pues bien, dado que, en las construcciones de (22) no hay Ensamble de papel temático externo, sobre el que puedan «descargarse» los papeles no asignados por parte de affidato, de semble y de parece, no puede tener lugar la operación bundling. De nuevo, esta argumentación es débil; ¿por qué, entonces, las construcciones correlativas con anáforas expresas sí son aceptables?

El problema, por cierto, es de aceptabilidad, precisamente, no de agramaticalidad, en el caso de la construcción de Ascenso, y de gramaticalidad en la construcción pasiva. La construcción pasiva, como la de (23a) con anáfora expresa, no tiene correlato en español, a menos que -no en todos los casos- se trate de una supuesta pasiva con el verbo estar, cf. las oraciones de (25):

(25) a. *Juan se ha sido confiado a sí mismo / Juan (le)ha sido confiado a Pedro durante su convalecencia.

b. ?Yo me he estado confiado a mí mismo toda la vida / Yo (le) he estado confiado a Pedro varios años.

c. ?Juan se ha estado confiado a su padrino en la adolescencia / Juan (le) ha estado confiado a su padrino en la adolescencia.

Pero lo cierto es que en oraciones como las de (25a, b) estamos ante un falso problema de incompatibilidad entre Reflexividad y Pasividad; los clíticos son correferenciales con el complemento dativo de «confiarle algo o alguien a alguien», de modo que no estamos ante una «pasiva reflexiva» y la agramaticalidad tiene que ver, en tal caso, con la interpretación semánticamente incongruente. Pero, por otra parte, la construcción pasiva es incompatible con la reflexividad porque las pasivas son construcciones inacusativas; su único argumento no puede ser receptor de un papel temático «externo», es un argumento interno, y su posición sintáctica prominente es derivada (probablemente a la posición de Especificador del STop (Sintagma Tópico), y naturalmente resulta incompatible -por la redundancia de papel temático- con un clítico correferencial, como se puede observar en (26):

(26) a. *Pedro se ha sido maltratado.

b. *María se fue designada coordinadora del Máster.

En (26), Pedro y María ostentarían el mismo papel temático que los clíticos con los que correfieren respectivamente ${ }^{32}$.

\footnotetext{
${ }^{32}$ La consideración de los clíticos reflejos como meras marcas de reflexividad (Grimshaw 1982) no cambia los hechos; la agramaticalidad sería un argumento a favor de la inergatividad de las construcciones reflexivas y en contra de su supuesta inacusatividad. (Si el se fuera una
} 
En cuanto a la primera construcción de (25c), el clítico no correfiere con el complemento dativo del verbo, pero sí con el sujeto Juan; la construcción es diferente de la $(25 \mathrm{~b})$, porque este $s e$ pertenece a la clase de los dativos considerados «superfluos» en la tradición gramatical; no posee carácter argumental y su papel tiene algo que ver con la «participación» o «interés» (en expresión de la gramática tradicional) del referente de Juan en la situación de «haber estado confiado a una persona», es decir, su valor está próximo al papel de un Benefactivo no exento de algún tipo de Agentividad. Por otro lado, la construcción (25a), con clítico correferencial con el complemento dativo del verbo, es, a mi juicio, una construcción posible, aunque en comunicación "marcada», es decir, de efecto pragmático determinado (de connotación determinada, contexto humorístico, irónico, etc.) ${ }^{33}$.

Por lo que respecta a las construcciones con el verbo de «Ascenso» por excelencia, parecer y sus correlatos afines en otras lenguas, no comparto el juicio de agramaticalidad. Se trata simplemente de emisiones no aceptables porque resultan informativamente ambiguas o imprecisas. Es obvio que si la razón de la supuesta agramaticalidad de Juan se parece inteligente o Jean se semble intelligent tiene que ver con el hecho de que "there is no merger of an external role, upon which the unassigned role of [...] semble ('seems') can be discharged», ¿cómo se explica que las proformas anafóricas explícitas decidan la gramaticalidad de las construcciones, si tenemos en cuenta que pueden coaparecer con el clítico? (cf.: tú rara vez te pareces buena a ti misma). Obsérvese, además, que los verbos de Ascenso, son naturalmente inacusativos. Su argumento -proposicional en cualquier caso- es un AI, y el sujeto sintáctico de la construcción sería derivado de su posición de argumento del predicado proposicional o clausal dominado por el verbo de «Ascenso", por lo que parecer, sembler, y semejantes no han de asignar papel temático al sujeto sintáctico Juan, Jean,...

Probablemente, la razón por la que no decimos naturalmente tú te pareces inteligente sin el doblado de la expresión anafórica es análoga a la que impide la naturalidad de tú te adoras ${ }^{34}$. Se trata, en cualquier caso, de verbos que precisan el refuerzo de las proformas anafóricas plenas porque su posible interpretación reflexiva resulta ser, de algún modo, «marcada»-cf. tú me pareces inteligente; tú le adoras (con objeto no refle-

categoría morfológica que, además de reducir el Caso estructural, absorbiera el papel externo de Agente, las construcciones pasivas no serían incompatibles con la reflexividad.) table:

${ }_{33}^{33}$ Así, una emisión como la de (a) en un contexto determinado parece claramente acep-

a) Te confío a mi hijo pequeño durante mi estancia fuera de España, y por mí no te preocupes, que ya me confío a mí mismo; de hecho me he sido confiado a mí mismo desde que tenía uso de razón.

${ }^{34}$ Obsérvese que en determinados contextos, tales emisiones son válidas aun sin la expresión anafórica plena: haciendo eso,̨tú te pareces una buena persona?; tú te estás adorando constantemente;... 
jo) - pero no constituyen emisiones imposibles porque no son ni agramaticales ni inaceptables.

\section{Reflexivización y Fases}

En el marco del denominado Programa Minimista, la derivación sintáctica por Fases ha proporcionado más de una propuesta ${ }^{35}$ de análisis descriptivo de las construcciones reflejas favorable a la caracterización del clítico $s e-y$ afines- como marca de «morfología reflexiva» exclusivamente $^{36}$. Tales propuestas se fundamentan en el supuesto de que la morfología reflexiva ( $e$ o clítico o morfema similar) satisface el requisito de saturación de la posición de un argumento en el estadio correspondiente a la primera Fase de derivación sintáctica, de modo que tal Fase pueda ser accesible a la interpretación semántica (por el sistema I-C). Pero lo cierto es que las argumentaciones aducidas, tanto empíricas como teoréticas, no resultan adecuadas, particularmente en lo que se refiere al concepto de «Fase». Presentaré a continuación una breve discusión sobre una propuesta reciente: Basilico 2005.

Adaptando ideas de Rivero y Sheppard 2003, Basilico 2005 propone que el clítico reflexivo -la morfología flexiva (MF) en general- «cierra existencialmente» el argumento simple del verbo, de modo que la posición no-saturada del argumento objeto en el sv no incumpla el requisito de que «todas las posiciones argumentales deben ser saturadas en la interfaz (de interpretación) semántica». Ahora bien, la saturación del argumento de $\mathrm{v}$-el argumento interno- puede satisfacerse por la «regla» de «Identificación Argumental» (Argument Identification) que Basilico propone como una extensión de la de «Identificación Eventiva» (Event Identification) establecida por Kratzer 1996. Según la propuesta de Kratzer, cuando el sv se ensambla con el svoz (VoiceP-el Sv del $\mathrm{PM}^{37}$-) el argumento eventivo no saturado asociado con el sv se identifica con el argumento eventivo no saturado asociado con el papel temático del pre-

\footnotetext{
${ }^{35}$ Véase, por ejemplo, Lidz 1996; Basilico 2005. Las propuestas, en general, consideran conjuntamente datos de construcciones «reflejas» (María se peina); «antipasivas» (María se confiesa) y «anticausativas» (el vaso se rompió); cualquiera de estos tipos de construcciones contiene la misma morfología reflexiva -aparece el mismo clítico (o, en otras lenguas, el mismo morfema libre o trabado) - que es requerida por la derivación por Fase.

${ }^{36}$ Particularmente en los últimos trabajos de Chomsky 2005, 2006 se precisa con mayor rigor este concepto de «Fase» como estadio de generación de objetos sintácticos (digamos, construcciones sintácticas) que se transfiere al Componente Intencional-Conceptual para recibir interpretación semántica.

${ }^{37}$ Nos referimos aquí al $v$ light o little en general, del Programa Minimista, sin la distinción entre $v^{*} \mathrm{y} v$, porque no es relevante a efectos de la discusión que se expone.
} 
dicado (p. ej. Agente) ${ }^{38}$. Pues bien, parafraseando a Basilico, la «Identificación Argumental» permitiría que el argumento no saturado de v se identifique con el argumento del $v$ (el Agente, p. ej.), de modo que solamente se necesitaría un argumento en la sintaxis para saturar dos argumentos semánticos. El sN ${ }^{39}$ introducido en la posición de Especificador de $\mathrm{S} v$ saturará los dos argumentos: el externo, en relación con $v$ y el argumento de v. Así que, con tal de que un argumento simple del verbo permanezca no saturado, es posible obtener una lectura reflexi$v^{40}{ }^{40}$ y no resulta sorprendente que no sea necesario ningún sN expreso en la sintaxis de las construcciones reflexivas. Pero la presencia del clítico reflexivo es necesaria, según Basilico, como consecuencia de la estructura de Fase de la oración ${ }^{41}$; puesto que los dos argumentos, el Interno y el Externo, son introducidos en Fases separadas (el primero en la Fase Sv y el segundo en la Fase $\mathrm{S} v$ ) -arguye el autor- el clítico permite saturar la posición del argumento simple -el objeto- de v. Sin el clítico/sufijo reflexivo, la entidad sintáctica Sv sería enviada a la interfaz semántica con el argumento simple de v no saturado antes de alcanzar la Fase siguiente, lo que incumpliría la condición mencionada de la interfaz semántica, que requiere que todas las posiciones de argumento individual estén saturadas, y, naturalmente, han de estarlo en cada Fase, porque, de no ser así, no habría posibilidad de convergencia.

Ahora bien, -concluye el autor- si la lectura reflexiva ha de obtenerse por el proceso de «Identificación Argumental» es necesario que el argumento de $\mathrm{v}$-el objeto- permanezca no-saturado, así que la solución a este dilema consiste en adoptar un proceso de existential disclosure (Dekker 1993), tal como ha sido adoptado por Rivero y Sheppard 2003 en contextos de impersonales clíticos. La «desactivación» del «cierre existencial» se produciría en la Fase Sv; el argumento Externo, habilitado por el $v$ light-que contiene en sí mismo el papel temático del predicado SV- suprimiría el cuantificador existencial, es decir, «desactivaría» el cierre existencial del argumento interno, permitiendo en la Fase S $v$ la aplicación de la «Identificación Argumental» para obtener la lectura reflexiva.

${ }^{38}$ La representación semántica de Event Identification sería así: $\lambda \mathrm{x} \lambda \mathrm{e}[\operatorname{Agent}(\mathrm{x})(\mathrm{e})] \quad \lambda \mathrm{e}[$ feed (the dog) $(\mathrm{e})] \rightarrow$ $\lambda \mathrm{x} \lambda \mathrm{e}[$ Agent $(\mathrm{x})(\mathrm{e})] \quad \& \quad[$ feed $($ the $\operatorname{dog})(\mathrm{e})]$

${ }^{39}$ Aunque está generalizado el símbolo SN, parece más adecuado optar por SD -Sintagma Nominal con Determinante- para esta posición en lenguas como el español, en las que los sujetos han de construirse por defecto con Determinante.

${ }^{40}$ En términos de Basilico: «as long as the single argument of the verb remains unsatured, a reflexive reading is possible».

${ }^{41}$ Textualmente observa Basilico 2005, p. 119: «I argue that the presence of the reflexive clitic/suffixis is actually a consequence of the phase structure of the clause and certain requeriments of the LF interface». 
La propuesta de Basilico presenta, a mi juicio, más de un aspecto discutible. En primer lugar, la «Identificación Eventiva» de Kratzer no puede hacerse extensiva a los argumentos «individuales», no eventivos, porque la motivación es distinta; la «Identificación Argumental» que propone Basilico tiene que ver con correferencialidad de argumentos, lo que no ocurre en el caso del argumento eventivo [e]. Por otro lado, si bien resulta sugerente la idea -no muy alejada de la de Reinhart y Siloni 2005- de que, por Identificación Argumental, un argumento sintáctico saturaría dos argumentos semánticos, lo cierto es que la no-saturación de la/una posición argumental de objeto no implica identificación de tal argumento con otro, como se ha observado anteriormente ${ }^{42}$.

La objeción más seria a la propuesta de Basilico tiene que ver con el concepto de Fase. Contrariamente a lo que el autor observa, Sv y sv no son Fases separadas porque sv no constituye nunca Fase de derivación, de modo que solamente $\mathrm{S}^{43}$ se determinaría como primera Fase de derivación y, consecuentemente, la motivación del clítico para saturar la posición argumental del argumento de v por «cierre existencial» dejaría de tener sentido respecto a la entidad sintáctica sv, puesto que tal entidad ya no «es enviada» como Fase de derivación a la interfaz semántica. Y, correlativamente, ya no es preciso recurrir a la operación siguiente de «desactivación» del cierre existencial en la Fase $\mathrm{S} v$, dado que esta sería la única Fase en la que se constituye la estructura temático-argumental completa ${ }^{44}$.

Ahora bien, si la «clave» de la interpretación reflexiva es la Identificación Argumental en función de la posición no saturada de un argumento (el de Objeto o argumento Interno) ¿por qué hay lenguas (la mayoría ${ }^{45}$ )

${ }^{42}$ Cf. p. ej., construcciones del tipo: María estuvo lavando y peinando toda la mañana (en la peluquería de su madre); etc. Por otro lado, el hecho de que existan construcciones con verbos transitivos y clíticos no reflejos sin los argumentos correlativos expresos, es decir, con las correspondientes posiciones argumentales no saturadas, «desautoriza» el proceso de 'Identificación Argumental', puesto que no se produce tal identificación de argumentos (cf.: Juan la lavó; te peinó muy bien).

${ }^{43}$ Recuérdese que, en los últimos trabajos de Chomsky, se distingue entre $v^{*} \mathrm{y} v$, pero solamente $\mathrm{S} v$ * es Fase; en la propuesta de Basilico, $\mathrm{S} v$ sería equivalente al actual $\mathrm{S} v$ *, puesto que el autor se basa en el primer texto de referencia sobre el concepto de 'Fase' (Chomsky 2001).

${ }^{44}$ Ocioso es observar que, en la misma Fase, la que corresponde al $\mathrm{S} v$ de Basilico, no se puede «activar» «cierre existencial» o saturación de un argumento para «desactivarlo» a continuación. Pero, curiosamente, el propio Basilico, en su propuesta, infiere que las construcciones reflexivas plantean dificultades en relación con la teoría de la Fase porque el argumento objeto debe tener la misma referencia que el sujeto, de modo que la interpretación del objeto depende de la del sujeto, pero, entonces, el objeto no puede ser interpretado antes del sujeto dentro de la Fase sv y como -en su propuesta- los dos argumentos son introducidos dentro de Fases separadas, se plantea un dilema. Hay que tener en cuenta que una vez que una Fase pasa a la interfaz Semántica no está disponible para la adición -ni supresión- de material alguno, ni sintáctico ni semántico, y la solución que el autor propone es arbitraria y ad hoc: lo relativo al «cierre existencial» queda exento de este requerimiento.

${ }^{45}$ Recuérdese que, en las oraciones reflejas de lenguas como el inglés, sí es posible considerar la adecuación de un cierto tipo de operación interpretativa como la de Identificación Argumental que Basilico propone. Pero la mayor parte de las lenguas observadas poseen marcadores fonéticamente visibles de Reflexividad. 
que cuentan con clíticos (o afijos) reflexivos? La razón es porque existen lenguas que codifican la concordancia de objeto (directo o indirecto). Es decir, los clíticos «argumentales»-y también los afijos de función paralela- son marcas de flexión que los núcleos verbales pueden ostentar en relación con la estructura argumental de objeto ${ }^{46}$. La presencia de este tipo de flexión garantiza la existencia de las posiciones argumentales correspondientes aunque tales posiciones no estén ocupadas por argumentos plenos, fonéticamente visibles, de modo que el argumento pleno es recuperable contextualmente con referencia disjunta o puede ser identificado sin dificultad por correferrencialidad con el argumento sujeto -el argumento «externo»-; este último caso es el de contextos de Reflexividad. Si estas reflexiones son válidas, no podemos aceptar, para las oraciones reflexivas, propuestas basadas en la consideración de que un argumento sintáctico satura dos argumentos semánticos. Se trata de una posición -la de objeto- no saturada por un argumento fonéticamente visible, no de un argumento no-saturado. (Nótese que cuando hablamos de «identificación» o de correferencialidad de argumentos, tenemos en cuenta necesariamente dos argumentos ${ }^{47}$ y la no-visibilidad o no accesibilidad fonética de un argumento no cancela su existencia).

\section{UNA NOTA SOBRE «RECIPROCALIZACIÓN»}

Por lo que respecta a otras operaciones de reducción de valencia sintáctica de un predicado (arity Operations), a saber, Reciprocalization, De-

\footnotetext{
${ }^{46}$ Según Anderson 2005, p. 239, los clíticos constituyen una forma de concordancia, que difiere de la concordancia verbal propiamente dicha, con morfemas desinentes flexivos, solamente porque su contenido funcional, gramatical, está morfológicamente realizado en forma libre, y no trabada. Con orientación similar, García Fernández 2008 considera, en relación con el español, que el verbo puede estar asociado con tres tipos de concordancia nominal: una en caso recto y dos en caso oblicuo. La primera la ostentan los morfemas flexivos de las formas finitas del verbo y la concordancia de objeto -de caso oblicuo- aparece expresada (con carácter obligatorio u opcional) en forma de clíticos -de acusativo o de dativo- y es compatible con las formas verbales finitas y no-finitas.

La concordancia de sujeto -en caso recto- refleja los rasgos de Número y Persona del sujeto, mientras que las concordancias de objeto -en caso oblicuo- refleja los rasgos de Número, Género, Persona, y Determinación del objeto (la de objeto dativo excluye el rasgo de Género). La concordancia en caso oblicuo puede poseer referencia disjunta con respecto a la concordancia de nominativo o puede correferir con esta.

${ }^{47}$ Los clíticos de concordancia de objeto pueden ser, como la flexión nominal, referenciales o no-referenciales; son referenciales, por ejemplo, los clíticos de (a) y la flexión de (b), y son no-referenciales los clíticos de (c) y la flexión de (d):

(a) se lo compré ayer ( $s e=$ a María; lo = un ordenador); Juan se afeitó esta mañana

(b) Llegasteis tarde (flexión de Persona Segunda y Número Plural)

(c) Nos arrepentimos; Juan se suicidó

(d) Ha nevado durante toda la noche (flexión defectiva de Persona Tercera y Número Singular).

Los clíticos reflejos que presentan las oraciones propiamente reflexivas son referenciales; en los demás casos son no-referenciales (véase García Fernández 2008).
} 
causativization y Saturation, es, sin duda, la primera, la que resulta más discutible en relación con la distinción «léxico-sintaxis».

Según Siloni 2001 existen predicados recíprocos léxicos y predicados recíprocos sintácticos. Dado que la predicación recíproca es subsidiaria de la predicación reflexiva, es esperable que el conjunto de lenguas que posee reflexividad léxica sea el mismo que presenta reciprocidad léxica; alternativamente, la reciprocidad sintáctica aparece en las mismas lenguas que ostentan la reflexividad en la sintaxis. Sin embargo, los datos no son concluyentes en todos los aspectos ${ }^{48}$.

Una de las pruebas más claras la constituye, como en la predicación reflexiva, la posibilidad de nominalización recíproca en las lenguas LEX, al parecer excluida para las lenguas SINT, tal como aducen Reinhart y Siloni 2005. Pero, una vez más, hay que insistir en el hecho de que tal posibilidad se sigue naturalmente de la inducción de reciprocidad -como ocurría con la reflexividad-mediante morfemas trabados específicos; es decir, si una lengua LEx, como el húngaro, dispone de afijos -infijosde «reciprocidad» que se adjuntan a una base verbal, sin dificultad se adjuntarán a una base -o raíz- nominal, como ya ha sido observado.

Ahora bien, la diferenciación paramétrica LEX-SINT respecto a la construcción con Predicativo o con predicados infinitivos no es clara para la Reciprocidad. Las predicaciones recíprocas que admiten Pvo o construcciones de infinitivo en las lenguas SINT no inducen necesariamente «reciprocidad». Así en (27), con Pvo, y en (28), con cláusula de infinitivo, la interpretación recíproca no es la única que se puede obtener; una lectura reflexiva distributiva es pefectamente posible en un contexto favorable:

(27) a. Juan y María se consideran muy inteligentes («el uno al otro / cada uno de ellos se considera inteligente a sí mismo»).

(28) a. Giovanni e Maria si sono visti danzare.

b. Juan y María se han visto bailar. («cada uno de ellos se ha visto bailar a sí mismo en un vídeo / el uno al otro»)

Por otra parte, las recíprocas de construcción «discontinua», mediante con (o with o similar) se consideran características de las lenguas LEX (Siloni 2001), como se infiere del contraste de (29):

(29) a. *Giovanni si e abbracciato con Maria (it.). *Juan se ha abrazado con María. Juan y María se han abrazado.

b. János csókol-óz-ott Mari-val (hung.). (Janos bes- REC-PAS Mari- con) Janos se besó con María. Janos y María se besaron.

${ }^{48}$ Los datos del italiano y del húngaro están tomados de Reinhart y Siloni 2005. 
Sin embargo, no son definitivos los datos respecto a la vinculación de las recíprocas discontinuas con el parámetro de lenguas LEx, que poseen la propiedad de Reciprocidad léxica. En primer lugar, porque la construcción discontinua propiamente dicha es un hecho sintáctico; en segundo lugar, porque las recíprocas sintácticas, digamos convencionales, no son incompatibles en muchos casos con recíprocas discontinuas, como puede verse en (30); y en tercer lugar, porque existen núcleos predicativos de significado recíproco que pueden realizarse en la sintaxis en construcción discontinua o no, pero no requieren un morfema del tipo se, como se puede comprobar en (31), de modo que, una vez más, los hechos lingüísticos sugieren la conveniencia de revisar la distinción paramétrica referida ${ }^{49}$.

(30) a. Pedro y María \{se pelearon / se enfadaron / se reconciliaron / se casaron...\}.

b. Pedro \{se peleó / se enfadó / se reconcilió / se casó / ...\} con María

(31) a. Pedro y María \{alternan en el oficio / concuerdan en la opinión / discuten constantemente / ...\}.

b. Pedro \{alterna / concuerda / discute / ... con María.

\section{Conclusiones}

Las observaciones desarrolladas en este trabajo permiten inferir dos aspectos relevantes de las construcciones reflexivas: en primer lugar, no son oraciones inergativas -ni inacusativas- sino transitivas con correferencialidad argumental, como sanciona la tradición gramatical. En segundo lugar, la Reflexividad es un fenómeno sintáctico, no léxico. No hay, para los núcleos verbales, codificación léxica de un rasgo del tipo [+reflexivo], en un sentido análogo a un tipo de rasgo como [+inacusativo] o [+transitivo], aunque, en función de su significado nocional, el verbo root -la «raíz» verbal- pueda ser susceptible de construcción con morfemas, clíticos o expresiones anafóricas plenas «reflejas» para derivar oraciones con interpretación reflexiva. Por lo tanto, la Reflexividad tiene que ver con interpretación de correferencialidad argumental inducida por morfemas específicos, por clíticos o por expresiones anafóricas adecuadas, pero, en cualquier caso, en el contexto oracional. Paralelamente, en este estudio se aducen razonamientos en contra de las propuestas que defienden la posibilidad de que un argumento sintáctico sature dos argumentos o dos papeles semánticos.

\footnotetext{
${ }^{49}$ Por razones de espacio, no proseguiré la discusión en relación con los demás tipos de construcciones afines a la Reflexividad, que son objeto de análisis en un trabajo posterior.
} 


\section{REFERENCIAS BIBLIOGRÁFICAS}

Anderson, S. A. (2005): Aspects of the theory of clitics, Oxford, Oxford University Press.

Arellano, B. (2008): «Los papeles temáticos en la teoría gramatical», ms., Universidad Complutense de Madrid.

BAsilico, D. (2005): «Antipassive, anticausative, and reflexive: Phases and reflexive morphology», en Bateman, L. y Ussery, C. (eds.), Proceedings of NELS 35 , pp. $115-125$.

Bouchard, D. (1984): On the content of empty categories, Dordrecht, Foris.

Burzio, L. (1986): Italian syntax. A government-binding approach, Dordrecht, Reidel.

Cano Aguilar, R. (1987): Estructuras sintácticas transitivas en el español actual, Madrid, Gredos.

Chierchia, G. (2004): "A semantics for unaccusatives and its consequences», en Alexiadou, A., Anagnostopoulou, E. y Everaert, M. (eds.), The unaccusativity puzzle, Oxford, Oxford University Press, pp. 22-59.

Сномsкy, N. (1982): Some concepts and consequences of the theory of government and binding, Cambridge, Mass., The мIт Press.

- (2001): «Derivation by phase», en Kenstowicz, M. (ed.), Ken Hale: A life in language, Cambridge, Mass., The мIт Press, pp. 1-52.

— (2005): «On phases», ms., MIT.

— (2006): «Approaching UG from below», ms., MIT.

Dekker, P. (1993): «Existential disclosure», Linguistics and Philosophy 16, pp. 561-588.

Fernández Leborans, M. J. (2005): Los sintagmas del español. II. El sintagma verbal y otros, Madrid, Arco-Libros.

García Fernández, L. (2008): «Correferencia parcial», ms. (próxima publicación en DICENDA, Madrid, Editorial de la Universidad Complutense).

Grimshaw, J. (1982): «On the lexical representation of Romance reflexive clitics», en Bresnan, J. (ed.), The mental representation of grammatical relations, Cambridge, Mass., The мIт Press, pp. 87-146.

- (1990): Argument structure, Cambridge, Mass., The Miт Press.

Gruber, J. S. (2000): "Thematic relations in syntax», en Baltin, M. y Collins, C. (eds.), The handbook of contemporary syntactic theory, Oxford, Blackwell.

KaYne, R. (1975): French syntax: The transformational cycle, Cambridge, Mass., The Mit Press.

Kratzer, A. (1996): «Severing the external Argument from its verb», en Rooryk, J. y Zaring, L. (eds.), Phrase Structure and the Lexicon, Dordrecht, Kluwer, pp. $109-137$.

Lidz, J. (1996): Dimensions of reflexivity, tesis doctoral, Universidad de Delaware.

Marantz, A. (1984): On the nature of grammatical relations, Cambridge, Mass., The MiT Press.

— (1997): «No escape from syntax: Don't try morphological analysis in the privacy of your own lexicon», en Dimitriadis, A., Siegel, L., Surek-Clark C., y Williams, A. (eds.), Proceedings of the $21^{\text {st }}$ Annual Penn linguistics colloquium, upenn working papers in linguistics 4, 2, pp. 201-225.

- (2005): «Rederived Generalizations», ms., New York University, citado por N. Chomsky 2006). 
Perlmutter, D. (1971): Deep and surface structure constraints in syntax, Nueva York, Holt, Rinehart y Winston.

Pesetsky, D. (1995): Zero syntax: Experiencers and cascades, Cambridge, Mass., The Mit Press.

Reinhart, T. y Siloni T. (2004): «Against the unaccusative analysis of reflexives», en Alexiadou, A., Anagnostopoulou E. y Everaert E. (eds.), The unaccusativiy puzzle, Oxford, Oxford University Press., pp. 159-180.

- (2005): «The lexicon-syntax parameter: Reflexivization and other arity operations», Linguistic Inquiry 36, pp. 389-436.

Rivero, M. L. y Shepard, M. (2003): «Indefinite reflexive clitics in Slavic: Polish and Slovenian», Natural language and linguistic theory 21, 1, pp. 89-155.

RIzzi, L. (1986): «Null objects in Italian and the theory of pro», Linguistic Inquiry 17 , pp. 501-557.

SÁEz, L. (2007): «Applicative phrases hosting accusative clitics», ms., Universidad Complutense de Madrid (próxima publicación en Selected Proceedings of GURT 2007, Georgetown University).

Siloni, T. (2001): «Reciprocal verbs», en Y. W. Faik (ed.), Proceedings of Israel Association for Theoretical Linguistics 17.

— (2002): «Active lexicon», Theoretical Linguistics 28, pp. 383-400.

Sportiche, D. (1998): Partitions and atoms of clause structure: Subjects, Agreement, Case and clitics, Nueva York, Routledge.

Vera Luján, A. (1996): «Esquemas oracionales ergativos reflexivos», Estudios Lingüisticos de la Universidad de Alicante 11, pp. 385-409.

Wunderlich, D. (2006): «Towards a structural typology of verb classes», en Wunderlich, D., Advances in the theory of the lexicon, Berlín, Mouton de Gruyter. 
